\title{
高强度预应力陶瓷的发展与探索
}

\author{
包亦望 ${ }^{1,2}$, 孙 熠 ${ }^{2}$, 旷峰华 ${ }^{1}$, 李月明 ${ }^{2}$, 万德田 ${ }^{1,2}$
}

(1. 中国建筑材料科学研究总院 绿色建筑材料国家重点实验室, 北京 $100024 ; 2$. 景德镇陶瓷大学 材料科学与工 程学院, 景德镇 333403)

摘 要：同步提升陶瓷材料强度及损伤容限是陶瓷发展的核心问题。一百多年前预应力技术大幅提高混凝土和玻璃 的弯曲强度，并在世界上广泛应用以来，预应力增强陶瓷材料的设计就成为一个百年梦想。本文总结了增强陶瓷的 国内外研究进展，并提出了全新的高强度高损伤容限复合陶瓷的预应力设计及模型，通过优化表面预应力设计， 在陶瓷构件表面能够形成一层高度压缩应力，从而阻止裂纹扩展，并抵消外加拉应力，达到提高陶瓷的强度及损 伤容限的目的。这种预应力设计理论和规程可应用到结构陶瓷、建筑陶瓷和日用陶瓷等不同领域，具有明显的通用 性和广泛性，且简单、经济，不受构件尺寸和形状的限制，因此极具应用前景。

关 键 词: 预应力设计; 陶瓷; 高强度; 损伤容限; 综述

中图分类号: TB332 文献标识码: A

\section{Development and Prospects of High Strength Pre-stressed Ceramics}

\author{
BAO Yiwang ${ }^{1,2},{\text { SUN } \mathrm{Yi}^{2}, \text { KUANG Fenghua }^{1} \text {, LI Yueming }}^{2}$, WAN Detian ${ }^{1,2}$ \\ (1. State Key Laboratory of Green Building Materials, China Building Materials Academy, Beijing 100024, China; 2. School of \\ Materials Science and Engineering, Jingdezhen Ceramic Institute, Jingdezhen 333403, China)
}

\begin{abstract}
It is a worldwide challenge to simultaneously improve the strength and damage tolerance of ceramics, which is a core issue in the development of ceramics and a goal pursued by materials scientists. While the pre-stressed design has been widely used to improve the strength of concrete and glass for over a century, a little progress has been made for ceramic materials. In this paper, the research progresses of ceramic reinforcement are summarized, and a new pre-stressed design and model of high strength and high damage tolerance composite ceramics are proposed. The novel design focuses on the generation of residual compressive stresses on the surface of ceramic components to inhibit crack initiation and growth, and offset the external tensile stress, which can be applied to different fields such as structural ceramics, architectural ceramics, domestic ceramics and so on. This simple and economical technique with no limitation of size and shape in ceramic components has great application prospects.
\end{abstract}

Key words: pre-stressed design; ceramics; strength; damage tolerance; review

陶瓷材料的强度、损伤容限等性能直接影响陶 瓷结构件的使用寿命和破坏失效概率。高性能结构 陶瓷具有耐高温、耐腐蚀和耐磨损等优异性能，但 存在脆性大和可靠性差的致命弱点 ${ }^{[1-3]}$, 严重制约 了陶瓷材料在航空航天、能源、机械和汽车等众多 领域的发展, 如陶瓷钢、陶瓷发动机 ${ }^{[4-5]}$ 等几十年前
的设想至今仍难以实现。因此，提高陶瓷构件的高 强度、高损伤容限等力学性能是关系到国防安全和 节能节源的重大问题，对结构陶瓷的发展及应用尤 为重要。同理, 在传统陶瓷领域, 提高传统陶瓷制品 的强度及损伤容限可以在承载能力不变的条件下减 小器具厚度，从而大幅度节约资源和能源，为我国

收稿日期：2019-07-17; 收到修改稿日期：2019-08-18

基金项目：国家重点研发计划(2017YFB0310401, 2018YFF0214502); 工信部技术推广项目(0714-EMTC-02-00573/6) National Key Research and Development Program (2017YFB0310401, 2018YFF0214502); Technical Promotion Project of Ministry of Industry and Information Technology (0714-EMTC-02-00573/6)

作者简介：包亦望(1957-), 男, 教授. E-mail: ywbao@ctc.ac.cn BAO Yiwang(1957-), male, professor. E-mail: ywbao@ctc.ac.cn 
传统陶瓷行业的节能、减排提供新思路, 并推动建 筑陶瓷行业的技术进步, 进一步巩固我国陶瓷领域 的技术优势，促进陶瓷行业的可持续发展、生态文 明建设及生态环境保护。但是, 高强度脆性材料通 常对表面损伤敏感, 其裂纹扩展阻力较低。因此, 如 何在提升陶瓷材料强度的同时提升损伤容限是陶瓷 可持续发展的核心问题。

一百多年前西方学者发明了预应力混凝土和预 应力玻璃(钢化玻璃), 大幅度提高了材料的抗折强 度, 并得到了广泛应用, 从而使陶瓷材料的预应力 增强设计成为研究热点。近百年来不断有学者通过 模拟预应力混凝土 ${ }^{[6]}$ 和钢化玻璃 ${ }^{[7-8]}$ 的形式来制备 预应力陶瓷。但是模拟混凝土在陶瓷里面埋钢筋显 然无法高温烧结 ${ }^{6}$, 只适用于低温陶土。而采用物理 钢化玻璃的方式制备预应力陶瓷，使陶瓷处于高温 软化态后快速降温, 虽然可以提高强度 ${ }^{[9-10]}$, 但是 二次高温并使陶瓷达到熔融态, 耗能耗时难以实用 化, 同时还带来热震损伤。此外, 模拟化学钢化玻璃 的方法对于表面含有玻璃相的陶瓷有一定的增强效 果 ${ }^{[11-12]}$, 但在经济和品种上受到限制。因此, 目前在 预应力陶瓷尝试性的研究中, 存在系统的理论研究 不足、缺乏数学几何表达和设计模型等问题, 本文总 结了当前增强陶瓷的国内外研究进展, 提出了全新的 高强度高损伤容限复合陶瓷的预应力设计及模型, 并 展望了预应力陶瓷未来的研究方向和应用前景。

\section{1 陶瓷增强技术}

陶瓷是典型的脆性材料, 具有抗压强、抗拉弱 的特性。陶瓷材料在实际使用过程中常常在应力较 低时发生脆性断裂, 且断裂强度比理论强度要低几个 数量级, 如 $\mathrm{Al}_{2} \mathrm{O}_{3}$ 陶瓷材料的理论强度约为 $38 \mathrm{GPa}^{[13]}$, 但实际获得的 $\mathrm{Al}_{2} \mathrm{O}_{3}$ 陶瓷材料的强度通常只有 0.4 0.5 GPa。Griffith 断裂理论 ${ }^{[14]}$ 表明, 陶瓷材料发 生脆性破坏(即断裂)的临界条件是在外界应力作用 下, 材料内部能够储存的弹性应变能等于裂纹扩展 需要克服的表面能, 如式(1)所示:

$$
\sigma=\left[\frac{2 E \gamma}{\pi c\left(1-v^{2}\right)}\right]^{1 / 2}
$$

式中: $\sigma$ 为断裂强度, $E$ 为弹性模量, $\gamma$ 为断裂表面能, $c$ 为缺陷尺寸, $v$ 为泊松比。在晶体结构稳定的情况 下, 材料泊松比 $(v)$ 一般为常数, 强度由弹性模量 $(E)$, 断裂表面能 $(\gamma)$ 及缺陷尺寸 $(c)$ 三个参数控制。其中 $E$ 是非结构敏感参数, $\gamma$ 与微观结构有关, 但单相材料 的微观结构对 $\gamma$ 的影响小, 唯一可以控制的是材料
中的微裂纹。根据断裂力学理论, 即使在均匀拉伸 应力条件下, 表面裂纹对断裂的影响仍然大于内部 裂纹。从本质上说，陶瓷的断裂大多源于表面主裂 纹的扩展, 因此, 提高陶瓷材料强度和损伤容限的 关键是提高陶瓷材料抵抗裂纹扩展的能力及减缓裂 纹尖端的应力集中, 尤其是表面微裂纹。目前, 提高 陶瓷强度的方法有细化晶粒、高密度及高纯化、引 入增强体和预应力增强。

\section{1 细化晶粒、高密度及高纯化}

为了消除缺陷，提高晶体的完整性，细、密、匀、 纯是当前陶瓷发展的一个重要方面 ${ }^{[15-17]}$ 。通过振荡 热压致密化烧结 ${ }^{[18-23]}$ (热压烧结、热压振荡烧结等) 等工艺途径能明显地改善强度。Han 等 ${ }^{[20]}$ 对比了无压 烧结(Pressureless sintering; PS)、热压烧结(Hot press sintering; HP)及压力振荡烧结(Oscillatory pressure sintering; OPS)三种不同烧结工艺对陶瓷强度的影响, 研究表明, 随着烧结工艺由 PS、HP 发展至 OPS, 氧化 锆陶瓷的抗弯强度分别由(856 \pm 95$) 、(1303 \pm 131)$ 增加 至(1455 \pm 99$) \mathrm{MPa}$ 。但是这种热压方式受到样品形状 和尺寸的限制。此外, 细化晶粒(亚微米或纳米晶) 能提高陶瓷强度, 晶粒尺寸越小, 材料强度越高, 满 足正 Hall-Petch 关系。但是, 当晶粒尺寸小到一定程度 后, 会出现反 Hall-Petch 关系 ${ }^{[24]}$ 。Hirata 等 ${ }^{[25]}$ 研究了晶 粒尺寸对 $\mathrm{SiC}$ 陶瓷微观结构及性能的影响, 其中 $\mathrm{M}$ 系 列 $(25 \mathrm{vol} \% 30 \mathrm{~nm}+75 \mathrm{vol} \% 800 \mathrm{~nm}) \mathrm{SiC}$ 陶瓷强度明显 高于 A 系列陶瓷 $(100 \mathrm{vol} \% 800 \mathrm{~nm}$ ), 而 $\mathrm{N}$ 系列陶瓷 $(25 \mathrm{vol} \% 30 \mathrm{~nm}+75 \mathrm{vol} \% 330 \mathrm{~nm})$ 由于晶粒太小, 强 度反而有所下降(图 1)。

\section{2 引入增强体}

根据长度与粒径的比值(长径比), 增强体可分 为颗粒、晶须及连续长纤维三种。一般颗粒的长径 比在 5 以下, 晶须的长径比为 $5 \sim 100$, 连续长纤维的 长径比远大于 $100^{[26]}$ 。增强体是通过裂纹尖端桥联、 颗粒钉扎、裂纹偏转、弥散强化和纤维拔出等机理 来提高材料的强度 ${ }^{[26]}$ 。引入方法有直接引入 ${ }^{[27-32]}$ 或 原位反应(In-situ reaction synthesis) ${ }^{[33-34] 。 G u o ~}$ 等 $^{[35]}$ 在 $\mathrm{SiC}$ 陶瓷中加入 $5 \mathrm{wt} \%$ 纳米 $\mathrm{TiN}$ 颗粒, 抗折强度从 $472 \mathrm{MPa}$ 提高到 $686.8 \mathrm{MPa}$ 。Ding 等 ${ }^{[36]}$ 研究了不同 体积分数的碳纤维 $(28 \mathrm{wt} \%$ 5 5 wt \%) 对 $\mathrm{SiC}$ 陶瓷密度、 开孔率和机械性能的影响, 结果如表 1 所示。Chen 等 ${ }^{[37]}$ 利用粉煤灰、铝矾土和高岭土等原料, 在 $1390{ }^{\circ} \mathrm{C}$ 条件 下制备高强度(190 MPa)、低密度 $\left(1.48 \mathrm{~g} \cdot \mathrm{cm}^{-3}\right)$ 自生网 状莫来石晶须增强陶瓷，并探讨了烧结温度对陶瓷 弯曲强度及酸溶解度的影响(图 2)。这些方法主要应 用在军工、航天等重要领域, 也会受到成本以及形 状尺寸的限制。 

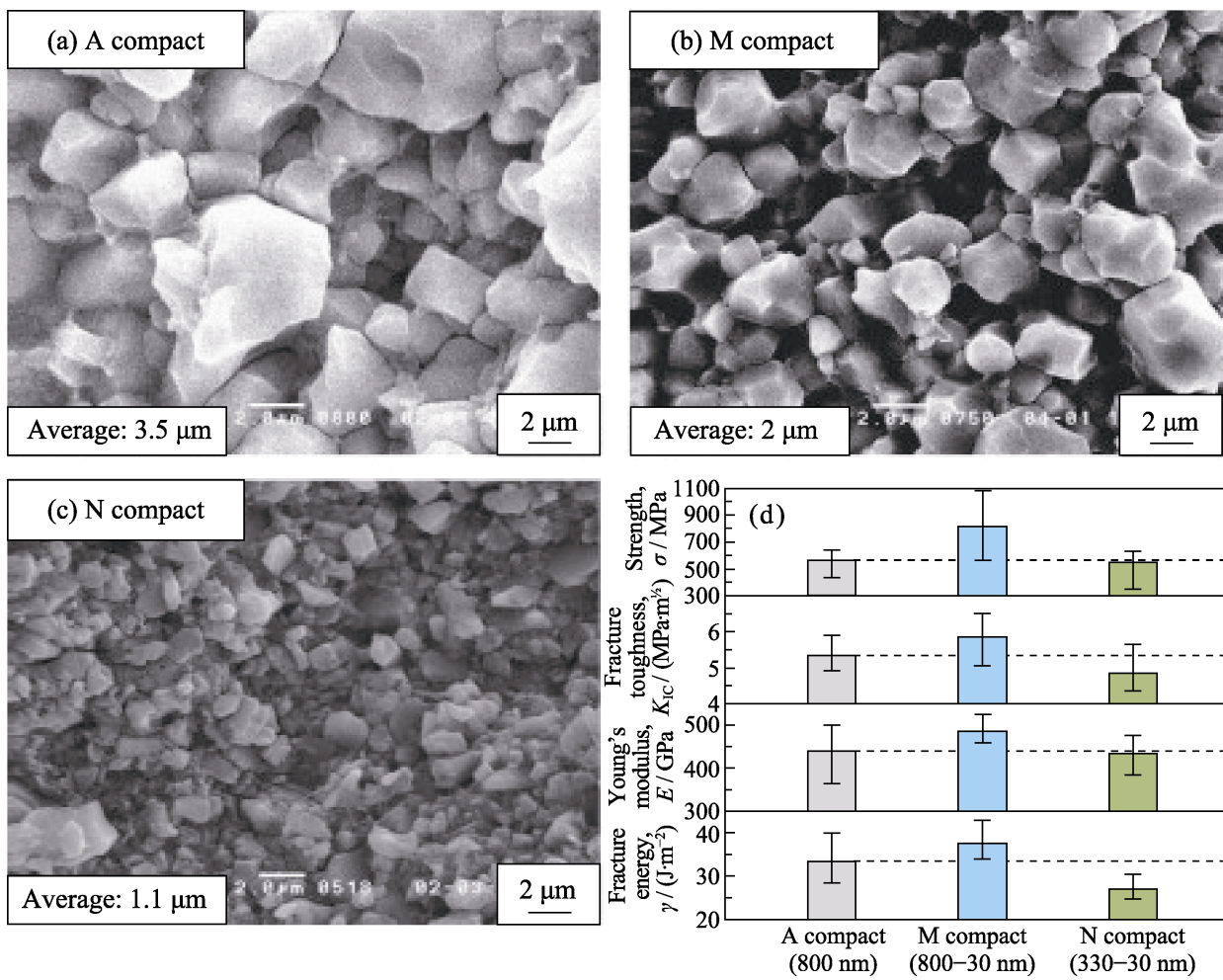

图 1 热压烧结不同系列 $\mathrm{SiC}$ 陶瓷的 $\mathrm{SEM}$ 照片 $(\mathrm{a} \sim \mathrm{c})$ 及力学性能 $(\mathrm{d})^{[25]}$

Fig. 1 Microstructures of A (a), M (b) and N (c) compacts, and mechanical properties of SiC compacts (d) ${ }^{[25]}$

表 1 不同体积分数纤维对 $\mathrm{SiC}$ 陶瓷机械性能的影响 ${ }^{[36]}$

Table 1 Mechanical properties of composites with different fiber contents ${ }^{[36]}$

\begin{tabular}{ccccc}
\hline Content of fiber & $28 \mathrm{vol} \%$ & $32 \mathrm{vol} \%$ & $43 \mathrm{vol} \%$ & $55 \mathrm{vol} \%$ \\
\hline Density $/\left(\mathrm{g} \cdot \mathrm{cm}^{-3}\right)$ & $(2.69 \pm 0.01)$ & $(2.55 \pm 0.02)$ & $(2.44 \pm 0.04)$ & $(2.27 \pm 0.03)$ \\
Open porosity/ \% & $(2.47 \pm 0.09)$ & $(2.95 \pm 0.01)$ & $(4.31 \pm 0.04)$ & $(4.85 \pm 0.03)$ \\
Bending stress $/ \mathrm{MPa}$ & $(198 \pm 37)$ & $(259 \pm 43)$ & $(325 \pm 35)$ & $(500 \pm 11)$ \\
\hline
\end{tabular}
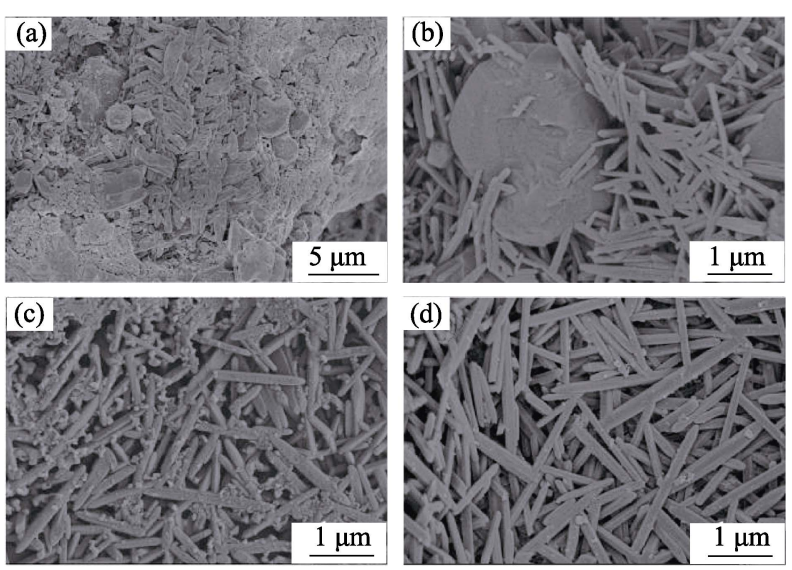

(e)

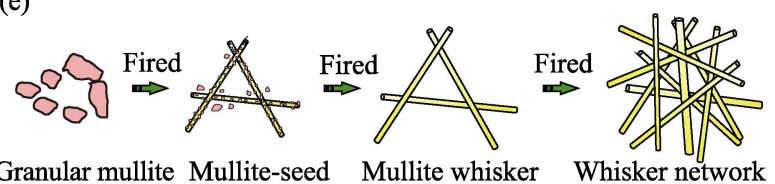

Granular mullite Mullite-seed Mullite whisker Whisker network

图 2 不同配方陶瓷的 SEM 照片(a d)及网状莫来石晶须形 成过程示意图 $(e)^{[37]}$

Fig. 2 SEM photomicrographs of different compositions (a-d) and formation process of the mullite whisker network (e $)^{[37]}$

(a) Sample 1; (b) Sample 2; (c) Sample 3; (d) Sample 4

\section{3 预应力增强}

众所周知, 现代工业建设中离不开混凝土和玻 璃。其中, 预应力混凝土由美国工程师 Jackson 于 1886 年首先提出, 但最初的运用并不成功。直到 1928 年, 法国工程师 Freyssinet 在对混凝土和钢材 性能进行大量研究和总结的基础上, 提出了预应力 混凝土必须采用高强钢材和高强混凝土以减少混凝 土收缩与徐变, 这一论断是预应力混凝土在理论上 的关键性突破 ${ }^{[38]}$ 。研究发现, 预应力混凝土结构的 抗裂性、刚度和承载力大大超过了钢筋混凝土结构, 不仅节约钢材, 改善结构功能, 解决了其他结构材 料难以解决的问题, 如增大房屋结构跨度、降低自 重等, 而且更是一种先进的结构形式 ${ }^{[39]}$ (图 3(a))。另 一个成功地应用预应力增强技术的是钢化玻璃, 1874 年由法国人发明, 采用急冷的方法制备钢化玻 璃, 并在英国申请了专利。钢化玻璃是通过在普通玻 璃表面形成一层预压应力，能够提高玻璃的强度 2 5 倍, 并提高其热稳定性和安全性能, 并于 20 世纪初 开始在全世界范围内得到全面的推广与普及 ${ }^{[7,8,40-42]}$ 
(图 3(b))。无论是在混凝土领域还是玻璃领域, 均采 用了一种宏观结构的预应力增强设计, 预先在材料 或构件中引入压应力以便抵消外加的拉应力, 从而 增加基体受张力而开裂的应变量, 达到提高材料断 裂强度、可靠性及耐久性的目的。如木桶, 在还没装 水之前采用铁䈨套紧桶壁, 便对木桶壁产生一个环向 的压应力, 若该压应力超过水的内压引起的拉应力, 木桶就不会开裂、漏水 ${ }^{[38]}$ (图 3(c))。此外, 利用熔融 金属包裹陶瓷复合形成高度紧凑约束的六面压缩应 力的预应力陶瓷，外表看似金属，能显著提高陶瓷 抗冲击及穿透能力, 可应用于防弹陶瓷领域 ${ }^{[33]}$ 。

目前，常见的人为预加应力的方法有：1)热韧 化处理即通过一定的加热、冷却制度在表面人为地 引入残余压应力。这种技术已被广泛应用于预应力 玻璃(钢化玻璃)行业 ${ }^{[7-8]}$ 和牙科陶瓷材料行业 ${ }^{[44]}$ 。 2)化学强化, 如离子置换法, 在基体升温时, 将材 料中小离子替换成大离子, 当材料冷却后, 则大离 子受到挤压形成钉扎效应, 给材料提供表层预压 力 $^{[11-12,41,45-46]}$ 。值得注意的是, 热韧化处理及化学强 化应用于玻璃中的应力分布特征(图 3(d)) 和增强效 果等方面也存在明显的不同；3)层状复合材料 (Laminated Composite), 是指复合材料中的增强相
分层铺叠，按相互平行的层面配置增强相，各层之 间通过基体材料连接, 主要利用增强层与陶瓷基体材 料的热膨胀系数的差异来提供预压应力。Song 等 ${ }^{[47]}$ 利用膨胀系数为 $8.1 \times 10^{-6} \mathrm{~K}^{-1}$ 的 $\mathrm{Al}_{2} \mathrm{O}_{3}$-石墨复合材 料与膨胀系数为 $8.5 \times 10^{-6} \mathrm{~K}^{-1}$ 的 $\mathrm{Al}_{2} \mathrm{O}_{3}$ 层制备了层 状复合材料, 结果显示, 层厚度、层数等因素能改变 材料的初始残余应力, 从而影响材料的弯曲强度和 断裂韧性等性能(图 4)。Qian 等 ${ }^{[48]}$ 利用低温共烧结 陶瓷(LTCC, low temperature co-fired ceramic)材料制 备 $404 \mathrm{MPa}$ 高强度层状复合材料, 可用于电子封装 (Electronic packaging)。

综上所述, 上述方法均能在一定程度上改善力 学性能, 但由于强化途径与方法不同, 机理与效果 也不同，很多技术受到陶瓷构件的形状和尺寸的限 制，同时，制备成本太大也难以普及到量大面广的 建筑卫生陶瓷和日用陶瓷领域。实际上，在经济实 用的陶瓷增强技术领域，国内外均已进入一种持续 无法突破的僵持阶段，通过采用各种增强剂制备的 复合材料，其强度也大多介于基体材料和增强材料 之间，且受到制备工艺、机器设备和生产成本等因 素的影响, 难以经济有效地工程化应用。此外, 预应 力增强技术虽然能够从工程化、实用化角度大 (a) Pre-stressed bridge and concrete

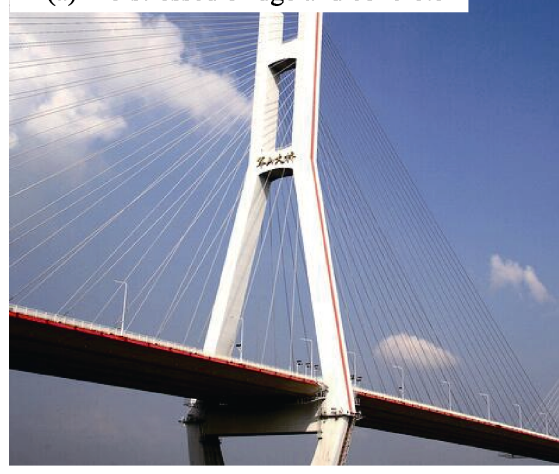

(c) Application of prestress technique

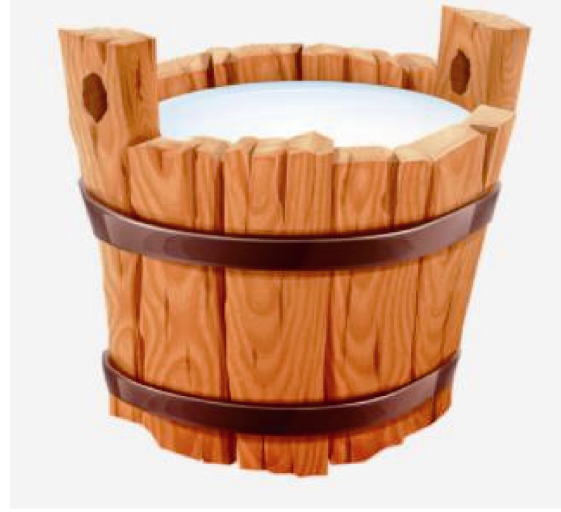

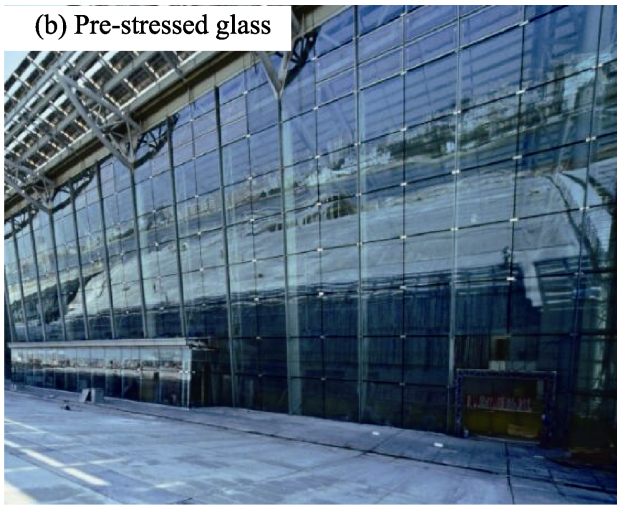

(d) Stress distribution curves

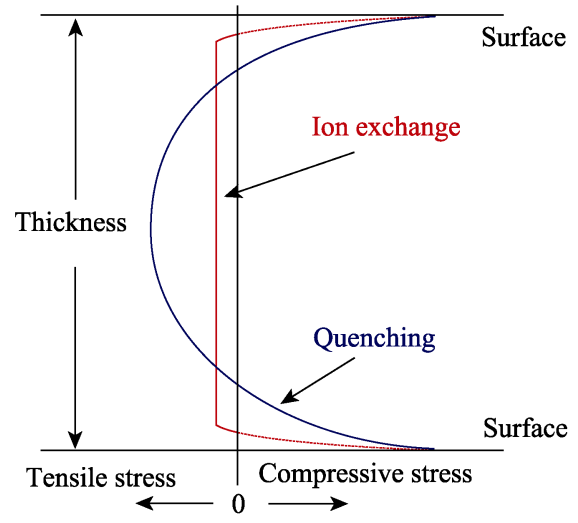

图 3 预应力技术在生活中的应用( $\mathrm{a} \sim \mathrm{c})$ 及不同增强方式在玻璃中的应力分布图(d)

Fig. 3 Application of prestressing technology (a-c) and stress distribution diagrams in glass (d) 


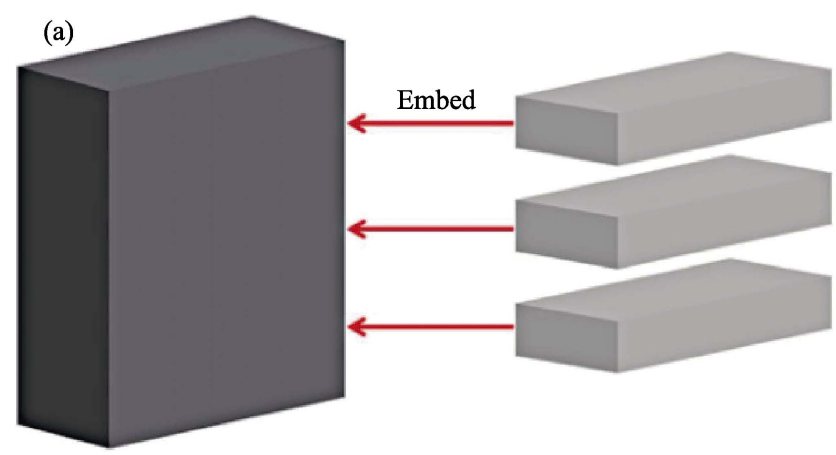

$\mathrm{Al}_{2} \mathrm{O}_{3}$-graphite composite

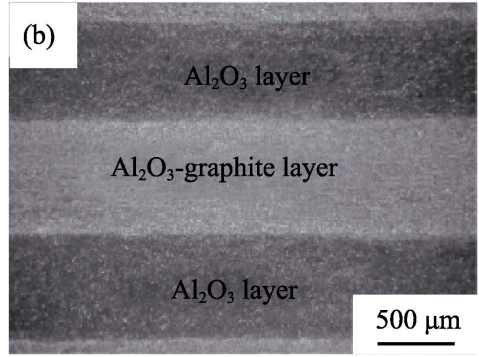

(e)

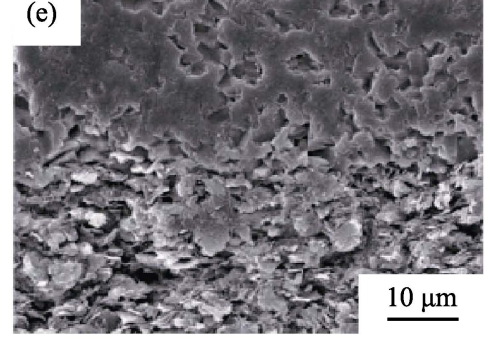

(h)

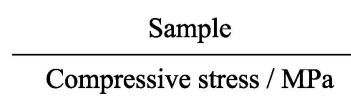

Tensile stress / MPa

Bending stress / MPa
$\mathrm{Al}_{2} \mathrm{O}_{3}$ layers
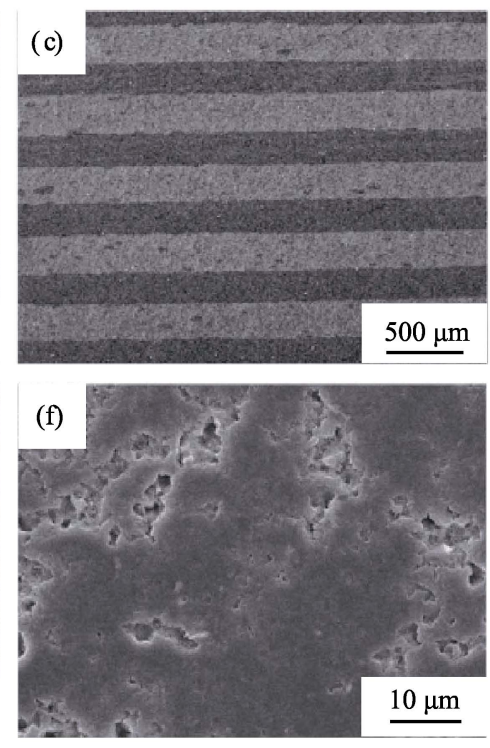
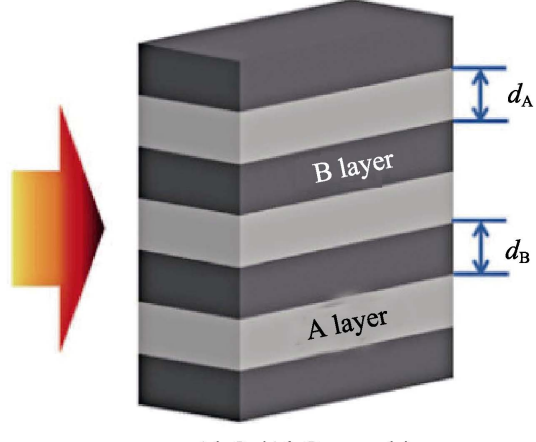

$\mathrm{Al}_{2} \mathrm{O}_{3} / \mathrm{Al}_{2} \mathrm{O}_{3}$-graphite laminated composite
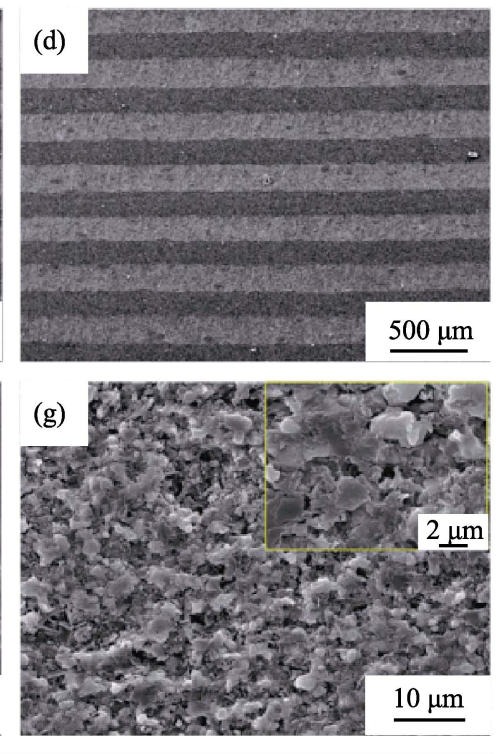

\begin{tabular}{ccccc} 
AG3 & AG5 & AG7 & AG9 & AG11 \\
-72 & -78 & -79 & -80 & -81 \\
108 & 97 & 93 & 90 & 89 \\
$(234 \pm 24)$ & $(292 \pm 16)$ & $(299 \pm 15)$ & $(304 \pm 12)$ & $(307 \pm 17)$ \\
\hline
\end{tabular}

图 4 层状复合材料设计及原理示意图(a), 不同参数(层厚、层数等)设计的陶瓷显微结构照片((b)AG3; (c)AG7;

(d)AG11; (e)复合材料界面; (f) $\mathrm{Al}_{2} \mathrm{O}_{3}$ 层的显微结构; ( g) $\mathrm{Al}_{2} \mathrm{O}_{3}$-石墨层的显微结构)及不同层状陶瓷的力学性能(h) ${ }^{[47]}$

Fig. 4 Schematic diagram of the laminated composites (a), microstructures of different structural composites $(\mathrm{b}-\mathrm{g})$ and mechanical properties of different laminate samples $(\mathrm{h})^{[47]}$

(b) AG3; (c)AG7; (d) AG11; (e) Interface of composite; (f) Microscopic structure of $\mathrm{Al}_{2} \mathrm{O}_{3}$ layer; (g) Microscopic structure of $\mathrm{Al}_{2} \mathrm{O}_{3}$-graphite layer

幅提升脆性材料的使用周期、可靠性及耐久性，但 由于陶瓷材料的复杂性，如何同步提升陶瓷构件的 强度及裂纹扩展阻力, 需从新的宏观设计和表面增 强方面进行探索和研究。

\section{2 高强度高损伤容限复合陶瓷的预应 力设计及模型}

2000 年, 中国最初始的预应力陶瓷是为了抗冲击 而提出的金属紧凑型约束预应力陶瓷(Pre-stressed ceramics $)^{[43]}$, 即利用金属熔体包裹陶瓷, 在冷却中 缓慢收缩, 形成对陶瓷的静水压力。既通过高温下
熔融铝合金对氧化铝陶瓷进行包覆，再冷却到常温 来获得预应力陶瓷样品。由于铝合金熔体的冷却收 缩会在陶瓷片表面形成压应力约束，从而导致陶瓷 内存在多向压应力和紧凑约束, 大大提高陶瓷的抗 冲击和抗穿甲能力(图 5)。但同时外部的金属也限制 了陶瓷的优点(如耐磨性, 耐高温, 耐腐蚀等 $)^{[49-50]}$ 。因 此，研发不含金属的预应力高强复合陶瓷，并不受 尺寸和形状的限制，从机理和模型上建立通用的预应 力设计规程是预应力增强的关键。由于陶瓷的破坏大 多是由表面拉应力引起的, 如果能使陶瓷构件表层形 成一层压应力, 就可以抵消外加拉应力, 阻止表面 裂纹的扩展(损伤容限), 制成高强度高损伤容限陶瓷。 

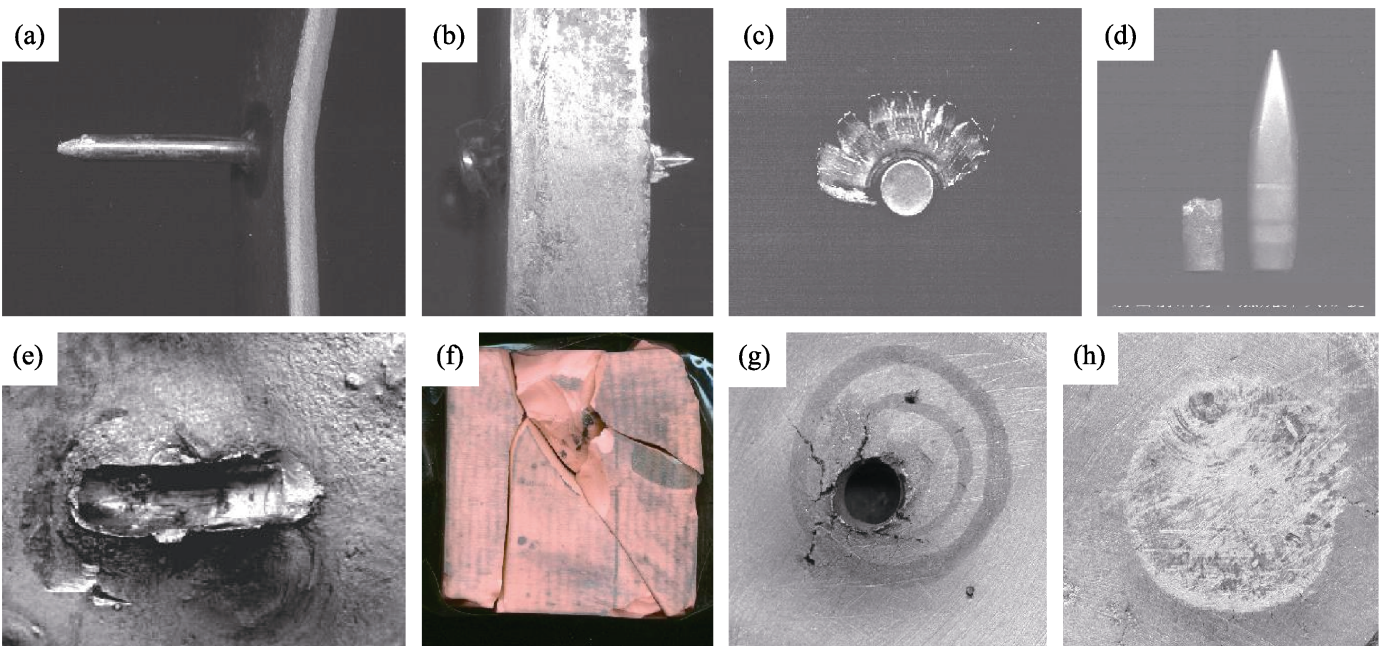

图 5 预应力陶瓷和无预应力陶瓷的抗冲击行为及抗穿甲对比试验 ${ }^{[50]}$

Fig. 5 Comparisons of impact resistance and armor piercing resistance between pre-stressed and unpre-stressed ceramics ${ }^{[50]}$ (a) Test of impact resistance performance with the nail gun, which could penetrate the steel plate with a thickness of $6 \mathrm{~mm}$; (b) $30 \mathrm{~mm}$-thick aluminum alloy plate penetrated by nail gun; (c) Mushroom-shaped fragments of pre-stressed composite materials fired by ordinary rifle bullets;

(d) Morphologies of the armor-piercing incendiary bullets before and after firing at pre-stressed composite materials; (e) Morphology of aluminum alloy pre-stressed ceramics after shooting; (f) Unpre-stressed ceramic materials being crushed and penetrated after shooting; (g) Frontal morphology of pre-stressed composite materials fired by armor-piercing incendiary bullets; (h) Reverse morphology of the pr-estressed composite materials fired by armor-piercing incendiary bullets, which could not penetrate the pre-stressed composite ceramics

为了在陶瓷等脆性材料表层形成一层压应力, 提出了涂层增强增韧陶瓷的方法。利用表层和基体 两种陶瓷之间膨胀系数和弹性模量的差异, 烧结后 使复合陶瓷构件的表面形成较强的压应力, 而内部 基体形成与之平衡的拉应力, 通过预应力计算和层 厚比优化设计, 实现整体应力平衡和能量均衡。通 常情况下, 陶瓷弯曲强度依赖于最大外加应力和表 面缺陷影响, 如果表面存在压缩预应力, 它制约裂 纹扩展并抵消外加拉应力, 从而提高强度; 反之, 表面存在拉伸预应力，它对表面裂纹有促进作用而 降低强度。为了实现表层压应力, 表层材料的膨胀 系数必须低于基体材料, 而弹性模量则越高越好。 按照这种方式制备的预应力陶瓷构件的残余应力分 布如图 6 所示。涂层法的前提条件是表层陶瓷必须 与基体陶瓷具有良好的匹配性和烧结相容性, 因此, 调配弹性模量和膨胀系数可以实现表面压缩残余应 力的形成, 而涂层中的残余应力满足式 $(2)^{[51]}$ :

$$
\sigma_{\mathrm{c}}=\left(\frac{S_{\mathrm{s}}}{S_{\mathrm{c}}}\right) \cdot\left\{1-\frac{\left[\frac{E_{\mathrm{s}} S_{\mathrm{s}}}{E_{\mathrm{c}} S_{\mathrm{c}}}+\frac{\alpha_{\mathrm{c}}}{\alpha_{\mathrm{s}}}\right]}{\left[1+\frac{E_{\mathrm{s}} S_{\mathrm{s}}}{E_{\mathrm{c}} S_{\mathrm{c}}}\right]}\right\} E_{\mathrm{s}} \cdot \alpha_{\mathrm{s}} \cdot \Delta T
$$

式中, $E_{\mathrm{s}}$ 为基体的弹性模量 $(\mathrm{GPa}), E_{\mathrm{c}}$ 为涂层的弹性 模量 (GPa), $S_{\mathrm{s}}$ 为基体的横截面积 $\left(\mathrm{mm}^{2}\right), S_{\mathrm{c}}$ 为涂层的 横截面积 $\left(\mathrm{mm}^{2}\right), \alpha_{\mathrm{s}}$ 为基体的膨胀系数 $\left(\mathrm{K}^{-1}\right), \alpha_{\mathrm{c}}$ 为 涂层的膨胀系数 $\left(\mathrm{K}^{-1}\right), \Delta T$ 为温度差 $(\mathrm{K})$ 。

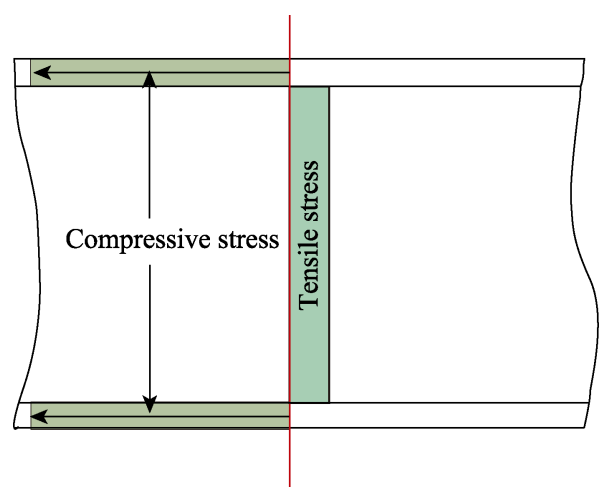

图 6 预应力复合陶瓷试样残余应力分布示意图

Fig. 6 Schematic of pre-stressing distribution in the ceramic composites

理论上陶瓷表层压缩预应力越大越好, 但是由 于表层预应力必须与内部的拉应力形成整体的力平 衡和能量均衡, 任何一个横截面的应力积分应为 零。所以表面压应力太大可能导致中间拉应力也随 之增大，使得破坏从内部拉应力区发生。因此，对于 两种成分确定的复相陶瓷，表层材料与基体材料之 间应存在一个最优的截面比, 该最优值可以通过构 件的尺寸、烧结温度、弹性模量以及本征强度等参 数计算得到。此外, 由于陶瓷表面存在压缩预应力, 也使得表面微裂纹处于压应力区而难以扩展，大大 提高陶瓷构件的损伤容限。这种预应力陶瓷增强设 计能广泛应用于结构陶瓷、日用陶瓷和建筑卫生陶 瓷等领域, 并能显著提高陶瓷弯曲强度, 采用无压 烧结不受形状和尺寸的限制。 

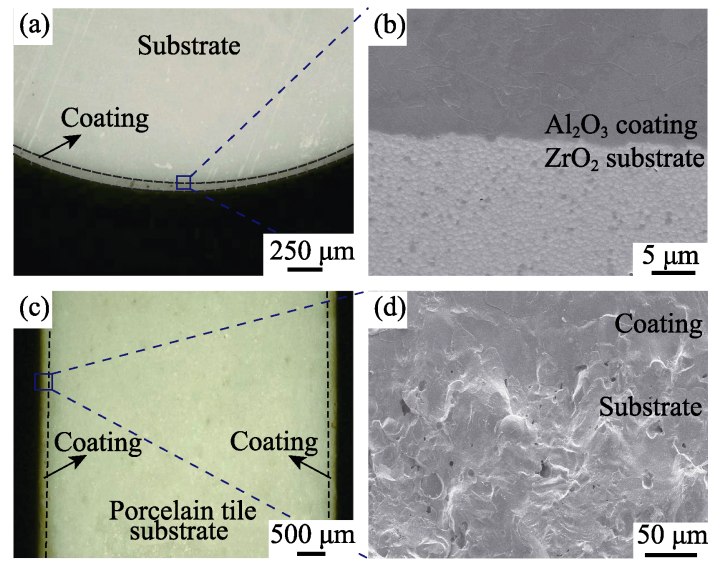

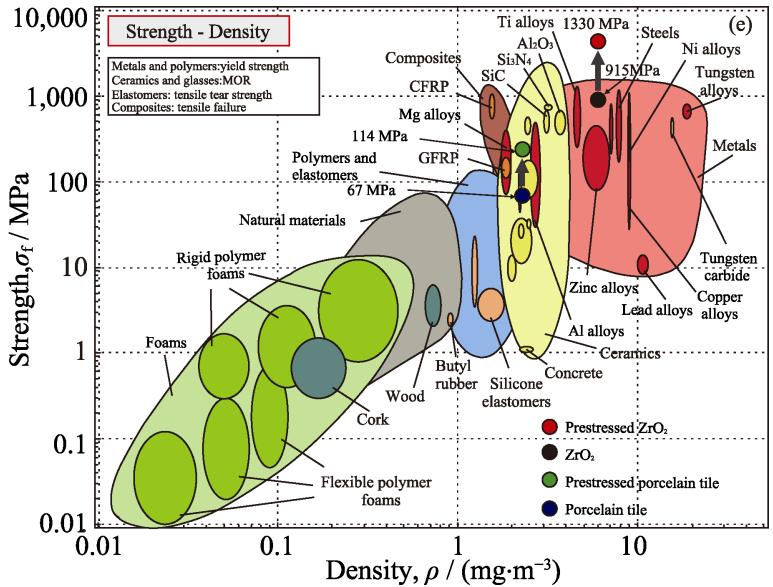

图 7 预应力陶瓷样品 $(\mathrm{a}, \mathrm{c})$ 、显微照片 $(\mathrm{b}, \mathrm{d})^{[51]}$ 及 Ashby 图解( $\left.\mathrm{e}\right)^{[52]}$

Fig. 7 Pre-stressed ceramics samples(a, c), SEM images $(b, d)^{[51]}$ and Ashby diagram(e $)^{[52]}$

$(a, b)$ Pre-stressed structural ceramics; (c, d) Pre-stressed architectural ceramics

依照上述模型，制备了两种预应力陶瓷，分别 为预应力结构陶瓷(图 7(a b)) 和预应力建筑陶瓷 (图 7(c d ) ), 其中预应力氧化锆陶瓷强度提升 45\%(从 $915 \mathrm{MPa}$ 提高至 $1330 \mathrm{MPa}$ ), 预应力建筑陶 瓷强度提升 $70 \%$ (从 $67 \mathrm{MPa}$ 提高至 $114 \mathrm{MPa}$ )。此外, 预应力氧化锆陶瓷的抗热震性能大幅提高。具体制备 流程与工艺见参考文献[51]。预应力氧化锆陶瓷在密 度不增加甚至下降的情况下, 弯曲强度高达 $1330 \mathrm{MPa}$, 显著高于 Ashby 图中最强工程陶瓷 ${ }^{[52]}$ (图 7(e))。

\section{3 预应力陶瓷应用前景及展望}

高强度高损伤容限预应力增强设计是陶瓷增强 领域中一种全新的思路, 有利于陶瓷向薄型化和轻 量化方向发展，同时利于产业节约资源和能源。此 外, 这种预应力设计采用无压烧结, 具有普遍性、低 成本、不受形状和尺寸的限制, 可工业化应用于结构 陶瓷，同时也可推广应用到量大面广的日用、建筑卫 生陶瓷领域乃至耐火材料领域。如果不考虑成本, 也 可以结合热压烧结和其他增强机制, 达到双重增强 的效果。

与预应力混凝土和钢化玻璃相似, 预应力陶瓷 也是一种构件, 而不是一种材料, 因为无论是哪种 预应力构件都不能进行机械加工, 否则破坏了预应 力的存在。预应力陶瓷可以应用到各种需要增强的 构件。如氧化铝陶瓷基片强度约 $400 \mathrm{MPa}$, 而氧化铝 涂层结合氧化锆基体的基片强度可达到 $1300 \mathrm{MPa}$ 。 建筑陶瓷墙地砖和日用陶瓷则可大幅度提高强度而 减少厚度, 实现传统陶瓷的薄型轻量化。

\section{参考文献:}

[1] KRSTIC Z. Silicon nitride: the engineering material of the future. $J$.
Mater. Sci., 2012, 47(2): 535-552.

[2] LAUNEY M E, RITCHIE R O. On the fracture toughness of advanced materials. Adv. Mater., 2009, 21(21): 2103-2110.

[3] GUO J K. Stress design of ceramic grain boundary. J. Inorg. Mater., 1995, 1(10): 27-31.

[4] GOHARDANI A S, GOHARDANI O. Ceramic engine considerations for future aerospace propulsion. Aircr. Eng. Aerosp. Tec., 2012, 84(2): 75-86.

[5] GARVIE R C, HANNINK R H, PASCOE R T. Ceramic steel? Nature, 1975, 258(5537): 703-704.

[6] JOHNSTON R D, CHIPMAN R D, KNAPP W J. Prestressed ceramics as a structural material. J. Am. Ceram. Soc., 1953, 36(4): 121-126.

[7] GREEN D J. Crack arrest and multiple cracking in glass through the use of designed residual stress profiles. Science, 1999, 283(5406): 1295-1297.

[8] WONDRACZEK L, MAURO J C, ECKERT J, et al. Towards ultrastrong glasses. Adv. Mater., 2011, 23(39): 4578-4586.

[9] INSLEY R H, BARCZAK V J. Thermal conditioning of polycrystalline alumina ceramics. J. Am. Ceram. Soc., 2006, 47(1): 1-4.

[10] CHEN L, WANG A, XIANGBO S, et al. Effect of surface heat transfer coefficient gradient on thermal shock failure of ceramic materials under rapid cooling condition. Ceram. Int., 2018, 44: 8992-8999.

[11] BARBI S, MUGONI C, MONTORSI M, et al. Chemical hardening of glazed porcelain tiles. J. Am. Ceram. Soc., 2019, 102: 2853-2862.

[12] SHAN Z, LIU J, SHI F, et al. A new strengthening theory for improving the fracture strength of lithium disilicate glass-ceramics by introducing $\mathrm{Rb}$ or $\mathrm{Cs}$ ions. J. Non-Cryst. Solids, 2018, 481: 479-485.

[13] RICHERSON D W, DEKKER M. Modern ceramic engineering, process, processing and use in design. New York, $2^{\text {nd }}$ edition revised, 1992: 21-33.

[14] GRIFFITH A A. The phenomena of rupture and flow in solids. Phil. Trans., 1921, 221(2): 163-198.

[15] 关振铎, 张中太, 焦金生. 无机材料物理性能. 北京: 清华大学 出版社, 1992: 92-93.

[16] MESSING G L, STEVENSON A J. Toward pore-free ceramics. Science, 2008, 322(5900): 383-384.

[17] ALFORD N M, BIRCHALL J D, KENDALL K. High-strength ceramics through colloidal control to remove defects. Nature, 1987, 330(6143): $51-53$.

[18] WANG W, FU Z, WANG H, et al. Influence of hot pressing sinter- 
ing temperature and time on microstructure and mechanical properties of $\mathrm{TiB}_{2}$ ceramics. J. Eur. Ceram. Soc., 2002, 22(7): 1045-1049.

[19] HUANG Y H, JIANG D L, ZHANG X F, et al. Enhancing toughness and strength of $\mathrm{SiC}$ ceramics with reduced graphene oxide by HP sintering. J. Eur. Ceram. Soc., 2018, 38(13): 4329-4337.

[20] HAN Y, LI S, ZHU T, et al. An oscillatory pressure sintering of zirconia powder: densification trajectories and mechanical properties. J. Am. Ceram. Soc., 2018, 101(5): 1824-1829.

[21] LI S, XIE Z P, XUE W J. Microstructure and mechanical properties of zirconia ceramics consolidated by a novel oscillatory pressure sintering. Ceram. Int., 2015, 41(8): 10281-10286.

[22] LI S, XIE Z P. Preparation of zirconia ceramics with high density and fine grains by oscillatory pressure sintering. J. Inorg. Mater., 2016, 31(2): 207-212.

[23] ZHU T, XIE Z, HAN Y, et al. Improved mechanical properties of $\mathrm{Al}_{2} \mathrm{O}_{3}-25 \mathrm{vol} \% \mathrm{SiC}_{\mathrm{w}}$ composites prepared by oscillatory pressure sintering. Ceram. Int., 2017, 43: 15437-15441.

[24] FURUKAWA, IWAHASHI, HORITA, et al. Structural evolution and the Hall-Petch relationship in an Al-Mg-Li-Zr alloy with ultra-fine grain size. Acta Mater, 1997, 45(11): 4751-4757.

[25] HIRATA Y, SUZUE N, MATSUNAGA N, et al. Particle size effect of starting $\mathrm{SiC}$ on processing, microstructures and mechanical properties of liquid phase-sintered SiC. J. Eur. Ceram. Soc., 2010, 30(9): 1945-1954.

[26] 刘维良, 喻佑华. 先进陶瓷工艺学. 武汉: 武汉理工大学出版 社, 2004: 636-664.

[27] ZhAN G D, KUNTZ J D, WAN J, et al. Single-wall carbon nanotubes as attractive toughening agents in alumina-based nanocomposites. Nat. Mater., 2003, 2(1): 38-42.

[28] PADTURE N P. Multifunctional composites of ceramics and single walled carbon nanotubes. Adv. Mater., 2010, 21(17): 1767-1770.

[29] OSTEEN K G, BRUNER K L, ONG D, et al. Single-crystal SiC nanowires with a thin carbon coating for stronger and tougher ceramic composites. Adv. Mater., 2010, 17(12): 1519-1523.

[30] ZHANG L, BEN Y, WU J, et al. Alumina assisted grain refinement and physical performance enhancement of yttria transparent ceramics by two-step sintering. Mat. Sci. Eng. A, 2017, 684: 466-469.

[31] FISHER E S, MANGHNANI M H, WANG J F, et al. Elastic properties of $\mathrm{Al}_{2} \mathrm{O}_{3}$ and $\mathrm{Si}_{3} \mathrm{~N}_{4}$ matrix composites with $\mathrm{SiC}$ whisker reinforcement. J. Am. Ceram. Soc., 1992, 75(4): 908-914.

[32] KHODAEIA M, YAGHOBIZADEHB O, ALHOSSEINI S H N, et al. The effect of oxide, carbide, nitride and boride additives on properties of pressureless sintered SiC: a review. J. Eur. Ceram. Soc., 2019, 39(7): 2215-2231.

[33] KUMAR S, SAIRAM K, SONBER J K, et al. Hot-pressing of $\mathrm{MoSi}_{2}$, reinforced $\mathrm{B}_{4} \mathrm{C}$ composites. Ceram. Int., 2014, 40(10): 16099-16105.

[34] YU H, CHEN Y, GUO X, et al. Study on mechanical properties of hot pressing sintered mullite- $\mathrm{ZrO}_{2}$ composites with finite element method. Ceram. Int., 2018, 44(7): 7509-7514.

[35] GUO X, YANG H, ZHANG L, et al. Sintering behavior, microstructure and mechanical properties of silicon carbide ceramics containing different nano-TiN additive. Ceram. Int., 2010, 36(1): $161-165$.

[36] DING Y, DONG S, ZHOU Q, et al. Preparation of C/SiC composites by hot pressing, using different $\mathrm{C}$ fiber content as reinforcement. J. Am. Ceram. Soc., 2006, 89(4): 1447-1449.

[37] CHEN X, LI T, REN Q, et al. Mullite whisker network reinforced ceramic with high strength and lightweight. J. Alloy Compd., 2017, 700: $37-42$.

[38] 李国平. 预应力混凝土结构设计原理. 北京: 人民交通出版社, 2000: $1-10$

[39] NAWY E. Reinforced concrete: a fundamental approach. Prentice-Hall, $2^{\text {nd }}$ edition revised, 1996: $1-15$.

[40] MAURO J C, PHILIP C S, VAUGHN D J, et al. Glass science in the united states: current status and future directions. Int. J. Appl. Glass. Sci., 2014, 5(1): 2-15.

[41] OLCOTT J S. Chemical strengthening of glass. Science, 1963, 140(3572): 1189-1193.

[42] CHAUDHRI M M, LIANGYI C. The catastrophic failure of thermally tempered glass caused by small-particle impact. Nature, 1986, 320(6057): 48-50.

[43] 包亦望, 郑元善, 苏盛彪, 等. 预应力陶瓷及其抗冲击与穿甲性 能. 材料导报, 2000, 14: 110-112.

[44] AURELIO I L, DORNELES L S, MAY L G. Extended glaze firing on ceramics for hard machining: crack healing, residual stresses, optical and microstructural aspects. Dent Mater, 2017, 33(2): 226-240.

[45] SHAN Z, LIU J, LIU M, et al. Surface strengthening of lithium disilicate glass-ceramic by ion-exchange using $\mathrm{Rb}, \mathrm{Cs}$ nitrates. Ceram. Int., 2018, 44: 12466-12471.

[46] DAL BÓ M, DOMINGUINI L, ZIMMER A, et al. Chemical tempering of porcelain tiles. Ceram. Int., 2016, 42: 15199-15202.

[47] SONG J, YANG H, BERMEJO R, et al. Enhanced thermal shock response of $\mathrm{Al}_{2} \mathrm{O}_{3}$-graphite composites through a layered architectural design. J. Am. Ceram. Soc., 2019, 102: 3673-3684.

[48] QIAN S, LIU F, MA M, et al. Mechanical strength enhancement of low temperature co-fired multilayer ceramic substrates by introducing residual stress. Ceram. Int., 2019, 45(8): 10982-10990.

[49] BAO Y, SU S, YANG J, et al. Pre-stressed ceramics and improvement of impact resistance. Mater. Lett., 2002, 57(2): 518-524.

[50] 包亦望. 先进陶瓷力学性能评价方法与技术. 北京：中国建材 工业出版社, 2017: 140-187.

[51] BAO Y, KUANG F, SUN Y, et al. A simple way to make pre-stressed ceramics with high strength. J. Materiomics., 2019, 5: $657-662$.

[52] ASHBY, M F. Materials Selection in Mechanical Design. Oxford, $4^{\text {th }}$ edition revised, 2011: 60-68. 